\title{
ESTUDIO PRELIMINAR DE LA AVIFAUNA ASOCIADA A \\ PARCHES DE Polylepis quadrijuga (Rosaceae) DEL PÁRAMO DE LA RUSIA, DUITAMA (BOYACÁ - COLOMBIA)
}

\section{LUZ ANDREA MENESES ORTEGÓN ${ }^{1}$, YIMY HERRERA- MARTÍNEZ ${ }^{2}$}

Recibido el 26 de agosto de 2012 y aprobado el 25 de enero de 2013

\section{RESUMEN}

Se realizó la caracterización preliminar de la avifauna presente en parches de Polylepis quadrijuga del Páramo de La Rusia en Duitama (Boyacá - Colombia), comparando dos épocas climáticas y tres parches durante 2007. Los parches se escogieron según la similitud fisionómica de la vegetación, interpretación de aerofotografías y reconocimiento en campo. En cada época y parche, se realizó el muestreo de la avifauna mediante observación directa en la mañana y la tarde y captura con redes de niebla. Con los datos obtenidos se determinó la riqueza de especies, y se realizó un análisis multivariado teniendo en cuenta las especies, la época y el parche muestreado. Se encontraron 34 especies de aves agrupadas en 29 géneros y 13 familias; de las cuales, Trochilidae y Emberizidae, fueron las de mayor riqueza. Siete especies de aves fueron comunes en los tres parches y el parche número 3 presentó el mayor número de especies (20), esto posiblemente debido a que este parche forma un ecotono con el bosque altoandino, es el de mayor tamaño (7 ha) y es atravesado por una quebrada. Los tres parches aportan especies de avifauna a la composición general del ecosistema.

\section{PALABRAS CLAVE}

Aves, Polylepis quadrijuga, diversidad, época, parche.

$$
\begin{aligned}
& \text { PRELIMINARY STUDY OF BIRD LIFE ASSOCIATED TO } \\
& \text { Polylepis quadrijuga (Rosaceae) PATCHES FROM "LA } \\
& \text { RUSIA", MOORLAND IN DUITAMA (BOYACÁ-COLOMBIA) }
\end{aligned}
$$

\footnotetext{
ABSTRACT

During 2007 a preliminary characterization of bird life present in the patches of Polylepis quadrijuga at "La Rusia" moorland, Duitama (Boyacá - Colombia), was carried out comparing two climatic seasons and three patches The patches were chosen according to their vegetation physiognomic similarity, interpretation of aerial photographs and field work. Sampling of bird life was carried out in each season and each patch through direct observation of birds in the morning and afternoon and through capture using mist-nets. Data obtained determined the bird species richness, and a multivariate analysis was made taking into consideration the species, the season and patch sampled.. Thirty-four species of birds grouped into 29 genera
} 
and 13 families were found from which Trochilidae and Emberizidae, were the richest. Six bird species were common to all three patches and patch number 3 showed the highest species richness (20), probably because this patch makes an ecotone with the high Andean forest, it is the largest ( 7 ha) and it is crossed by a stream. The three patches provide bird species to the overall ecosystem composition.

\section{KEY WORDS}

Birds, Polylepis quadrijuga, diversity, season, patch.

\section{INTRODUCCIÓN}

El género Polylepis (coloradito) al parecer evolucionó simultáneamente con el páramo que es un ambiente tropical frío, único y diferente a los ambientes de otras latitudes como el alpino templado y el ártico (Kerr, 2004). Polylepis se distribuye desde Argentina hasta Venezuela y forma desde rodales restringidos a zonas escarpadas y abrigadas de los páramos, hasta verdaderos bosques (Tarifa \& Yensen, 2001).

En Colombia se han registrado 2 especies del género Polylepis: $P$. sericea en la cordillera Central y $P$. quadrijuga en las cordilleras Oriental y Occidental (Fjeldså et al., 1996; Balderrama \& Ramírez, 2001; Valderrama \& Verhelst, 2006; Fundación PRO-AVES \& American Bird Conservancy, 2007). Estos bosques se encuentran amenazados en Colombia y se cree que se ha perdido cerca del $80 \%$ de su cobertura, debido a la creciente presión humana a través de la expansión de la frontera agrícola, las prácticas ganaderas, las quemas y la extracción de leña; lo que conlleva a la fragmentación y discontinuidad entre parches, afectando su estructura, extensión y composición específica (Venero \& Macedo, 1983; Fjeldså et al., 1996; Etter \& Villa, 2000; Fjeldså \& Kessler, 2004).

El corredor de conservación La Rusia - Guantiva - Iguaque presenta fragmentos aislados de Polylepis, lo que dificulta los procesos ecológicos que requieren realizar las poblaciones silvestres tanto de fauna como de flora asociada para su supervivencia (Rangel-Ch. \& Arellano, 2006), por lo que se ha clasificado como de alta prioridad de conservación (Hjarsen, 1997; Navarro, Molina \& De la Barra, 2005; Kessler, 2006). Estos bosques cumplen un rol central en la ecología altoandina, sirviendo de hábitat de especies animales, como depósitos de agua, protegiendo el suelo de la erosión y como fuente de recursos para los habitantes locales (Kessler, 2006).

Colombia posee la mayor diversidad de aves del mundo con 1897 especies descritas hasta el momento (Salaman, Donegan \& Caro, 2009; Donegan et al, 2012), que viven en una gran variedad de hábitats, de estas, aproximadamente 50 se han 
registrado en bosques de Polylepis. Las aves pueden ser de amplia o escasa distribución, que usan los bordes de estos bosques para anidar o descansar (Fjeldså \& Kessler, 2004), también se encuentran especies raras, casi endémicas y amenazadas (Fjeldså et al., 1996; Fjeldså, Lambin \& Mertens, 1999; Balderrama \& Ramírez, 2001; Fjeldså, 2002; Herzog, Soria \& Matthysen, 2003; Valderrama \& Verhelst, 2006).

En Colombia son escasos los estudios sobre la importancia de los parches de Polylepis para la diversidad biológica del páramo, especialmente de aves. El objetivo del presente trabajo fue realizar un estudio preliminar de la diversidad de aves asociada a parches de Polylepis quadrijuga del Páramo de La Rusia, Duitama (Boyacá - Colombia), en dos épocas climáticas del año 2007.

\section{MATERIALES Y MÉTODOS}

\section{Área de estudio}

El Páramo de La Rusia hace parte del Corredor de Conservación de Páramo y Bosque Altoandino Guantiva - La Rusia - Iguaque. Presenta una extensión territorial de $187 \mathrm{~km}^{2}$ ocupando los municipios de Duitama y Paipa (Boyacá Colombia), con elevaciones entre los 2900 y $3800 \mathrm{msnm}$ (Rangel-Ch., 2000), temperatura promedio de $9,78{ }^{\circ} \mathrm{C}$, precipitación media de $1200 \mathrm{~mm}$ año ${ }^{-1}$ y humedad relativa de 83\% (estación de Andalucía a 3265 m, estación Antena de TV La Rusia a 3650 m) (IDEAM, 2007). Geográficamente está ubicado entre los $5^{\circ} 51^{\prime}$ - $6^{\circ} 04^{\prime}$ Norte y $73^{\circ} 10^{\prime}-73^{\circ} 03^{\prime}$ Oeste.

Se escogieron tres parches de coloradito distanciados entre sí 2 $\mathrm{km}$, con el propósito de obtener una mayor visión de la diversidad de avifauna de este ecosistema. Los parches fueron seleccionados con base en interpretación de aerofotografías y salidas de reconocimiento, teniendo en cuenta su similitud fisionómica, la proximidad entre ellos y la facilidad de acceso. Los parches 1 y 2 se ubican en la vereda Peña Negra circundados por una matriz paramuna con áreas de 4 y 3 ha, pendientes de 11 y $13 \%$ y altitud de 3772 y 3672 m, respectivamente. El parche 3 se encuentra ubicado en la vereda Santa Bárbara contigua a la vereda Peña Negra, con un área de 7 ha, conforma un ecotono en el borde superior del Bosque Altoandino, tiene una pendiente de $22 \%$ y es atravesado por la quebrada Micaela y la carretera principal que conduce al municipio de Charalá. Cada uno de los parches fue geoposicionado y se estableció su altitud con GPS Mobile Mapper.

\section{Muestreo de avifauna}

Se realizó un muestreo de 5 días en cada época climática (lluvia y sequía) en cada parche de Polylepis, con el fin de 
realizar comparaciones entre parches y épocas. La caracterización de la avifauna se realizó a través de transectos de observación y captura con redes de niebla. Para los transectos se realizaron recorridos lineales de $100 \mathrm{~m}$ durante tres días, desde las 06:30 a las 11:00 h y de las 14:00 a las 17:00 h, recorriendo el parche en su interior y exterior (Stiles \& Bohórquez, 2000) para un total de 90 unidades muestrales. Las capturas con redes de niebla se realizaron instalando 10 redes de $36 \mathrm{~mm}$ de malla, 2,5 $\mathrm{m}$ de ancho y $10 \mathrm{~m}$ de longitud durante dos días. Las redes permanecieron abiertas desde las 6:30 a 10:30 h y fueron revisadas cada 30 minutos en el mismo orden en que se colocaron, para un total de $160 \mathrm{~h} / \mathrm{red}$ en cada parche (Ralph et al., 1996; Stiles \& Rosselli, 1998). En las aves capturadas se tomaron medidas morfométricas y registro fotográfico, con lo cual se realizó confirmación y determinación de especies. Seguido a la toma de registros, las aves fueron liberadas. La determinación taxonómica en campo se realizó utilizando la Guía de Aves de Colombia (Hilty \& Brown, 1986) y el Listado de Aves de Colombia de Salaman, Donegan y Caro (2009).

\section{Análisis de datos}

Se determinó la riqueza de especies, la abundancia, expresada en número de individuos de cada especie y la diversidad beta utilizando el índice de complementariedad (IC) desarrollado por Colwell y Coddington (1994). Los parches fueron comparados por escalado multidimensional no paramétrico (nMDS) (Kruskal, 1964) y Análisis Canónico de Coordenadas Principales (CAP) (Anderson \& Robinson, 2003; Anderson \& Willis, 2003) sobre los datos de abundancia de las especies detectadas por observación directa, estudiados en los 3 parches de $P$. quadrijuga. Se emplearon 999 reiteraciones para obtener la configuración bidimensional con menor estrés. Las bajas abundancias observadas no requirieron ningún tipo de transformación. En las ordenaciones se utilizaron similitudes de Bray-Curtis (Bray \& Curtis, 1957) entre transectos y parches.

Con los datos de disimilaridad se realizó el análisis SIMPER (Warwick, Clarke \& Saharsono, 1990; Clarke, 1993), para establecer las especies que más contribuyen a las diferencias entre parches. Las especies fueron registradas según el parche en el que se encontraron, para determinar especies comunes y exclusivas y así poder identificar la importancia de cada parche al aporte general de especies de aves de $P$. quadrijuga. Los análisis se realizaron utilizando los programas Primer V. 5.0 y CAP, este último desarrollado en FORTRAN (Anderson, 2004). 


\section{RESULTADOS}

La abundancia de aves de los tres parches de $P$. quadrijuga en el Páramo de La Rusia fue de 261 individuos y la composición incluyó 34 especies, las cuales se agruparon en 29 géneros, 13 familias y 3 órdenes. De estas, las familias Trochilidae con ocho especies y Emberizidae con tres especies, fueron las de mayor riqueza; esta dominancia fue homogénea para los tres parches de bosque (Figura 1).

En cuanto a riqueza, el parche 3 presentó 20 especies, el parche 2, 19 especies y el parche 1 presentó 18 especies de aves. El índice de complementariedad fue relativamente alto entre parches (P1-P2: 57,7; P1-P3: 72,4; P2-P3: 69,0) lo que indica gran diferencia entre ellos en cuanto a la composición de la avifauna; con solo siete especies comunes a los tres parches y nueve especies a dos; en tanto que 18 especies estuvieron presentes en uno solo de los tres parches (Tabla 1).

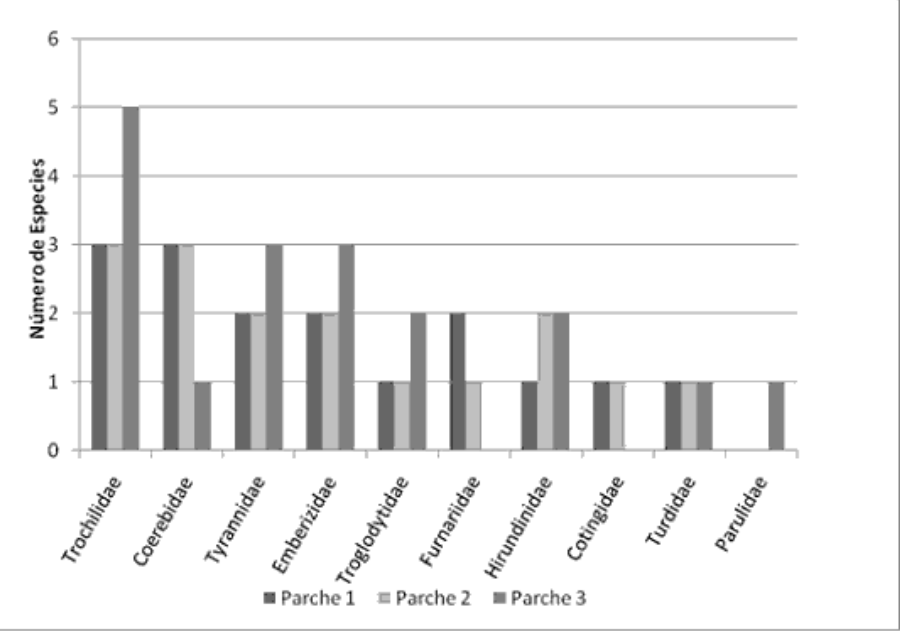

Figura 1. Riqueza de aves registrada en tres parches de $P$. quadrijuga del Páramo de La Rusia (Duitama, Boyacá) durante 2007 
Tabla 1. Presencia de aves en diferentes parches de P. quadrijuga del Páramo de La Rusia (Duitama, Boyacá) durante 2007

\begin{tabular}{|c|c|c|c|}
\hline Especie & Parche 1 & Parche 2 & Parche 3 \\
\hline Aglaeactis/cupripennis & $\bar{X}$ & & $\mathrm{X}$ \\
\hline Ampelion rubrocristatus & & $\mathrm{X}$ & \\
\hline Anisognathus igniventris & $\mathrm{X}$ & $\mathrm{X}$ & $\mathrm{X}$ \\
\hline Catamenia homochroa & & & $\mathrm{X}$ \\
\hline Cinnycerthia unirufa & & $\mathrm{X}$ & $\mathrm{X}$ \\
\hline Conirostrum rufum & $\mathrm{X}$ & $\mathrm{X}$ & \\
\hline Digiossa humeralis aterrima & & $\mathrm{X}$ & \\
\hline Digiossa humeralis humeralis & $\bar{X}$ & $\mathrm{X}$ & \\
\hline Diglossa lafresnayii & $\mathrm{X}$ & & $\mathrm{X}$ \\
\hline Eriocnemis mosquera & & & $\mathrm{X}$ \\
\hline Eriocnemis vestitus & & $\mathrm{X}$ & $\mathrm{X}$ \\
\hline Gallinago nobilis & $\bar{X}$ & $\mathrm{X}$ & \\
\hline Grallaria rufula & & $\mathrm{X}$ & \\
\hline Lafresnaya lafresnayi & & & $\bar{X}$ \\
\hline Leptasthenura andicola & $\mathrm{X}$ & & \\
\hline Lesbia victoriae & & $\mathrm{X}$ & \\
\hline Lipaugus fuscocinereus & $\bar{X}$ & & \\
\hline Margarornis squamiger & $\mathrm{X}$ & & \\
\hline Mecocerculus leucophrys & $\mathrm{X}$ & $\mathrm{X}$ & $\mathrm{X}$ \\
\hline Metallura tyrianthina & $\mathrm{X}$ & $\mathrm{X}$ & $\mathrm{X}$ \\
\hline Muscisaxicola alpinus & & & $\mathrm{X}$ \\
\hline Myioborus omatus & & & $\mathrm{X}$ \\
\hline Notiochelidon cyanoleuca & & $\mathrm{X}$ & $\mathrm{X}$ \\
\hline Notiochelidon murina & $\mathrm{X}$ & $\mathrm{X}$ & $\mathrm{X}$ \\
\hline Ochthoeca fumicolor & $\bar{X}$ & $\bar{X}$ & \\
\hline Oxypogon guerinii & $\mathrm{X}$ & & \\
\hline Phrygilus unicolor & $\mathrm{X}$ & $\mathrm{X}$ & $\mathrm{X}$ \\
\hline Ramphomicron microrhynchum & & & $\mathrm{X}$ \\
\hline Schizoeaca fuliginosa & & $\mathrm{X}$ & \\
\hline Carduelis spinescens & $\mathrm{X}$ & $\mathrm{X}$ & $\mathrm{X}$ \\
\hline Troglodytes aèdon & $\mathrm{X}$ & & $\mathrm{X}$ \\
\hline Trogiodytes solstitialis & & & $\mathrm{X}$ \\
\hline Turdus fuscater & $\mathrm{X}$ & $\mathrm{X}$ & $\mathrm{X}$ \\
\hline Mecocerculus sp & & & $\mathrm{X}$ \\
\hline
\end{tabular}

El análisis nMDS no discriminó los ensamblajes de aves por época, indicando que aparentemente los principales gradientes de variación no se ven afectados por la lluvia o la sequía. El resultado arrojado por el CAP es unidimensional y no detectó diferencias significativas entre los ensamblajes de aves por épocas climáticas $(p=0,07)$, pero el valor de $p$ podría considerarse secundariamente significativo. Las ordenaciones nMDS traslaparon las muestras procedentes de los tres parches de bosque de $P$. quadrijuga, en 999 reiteraciones aplicadas (Figura 2), indicando que aparentemente los principales gradientes de variación en la estructura de los ensamblajes de la avifauna, no respondieron al tipo de parche estudiado; el valor de estrés de la ordenación $(0,01)$ fue muy confiable. El CAP para los tres parches con el mismo número de reiteraciones (999) (Figura 2), reveló diferencias en los ensamblajes entre parches $(p=0,001)$ con base en los 12 ejes del Análisis de Coordenadas Principales (PCoA) que explicaron el $58 \%$ de la variación total, en dos ejes canónicos. El eje 1 separó a la izquierda los ensamblajes del parche 1 de los otros dos parches $(\delta 2=0,5)$, mientras que el eje 2 separó abajo los ensamblajes del parche 3 del resto $(\delta 2=0,3)$. Los porcentajes de reclasificación correcta para los transectos fueron de 52,58 y $65 \%$ para los parches 1, 2 y 3, respectivamente. Esta diferencia se debe a que nMDS es una ordenación no contrastante, es decir es una combinación lineal de las 
variables que minimiza la variación entre los puntos estudiados, mientras que CAP es una ordenación contrastante, en la que la ordenación lineal maximiza la variación dentro y entre grupos (Anderson \& Willis, 2003; Anderson, 2004), para este caso, las especies exclusivas son las que permiten la separación entre parches por CAP.

Tabla 2. Especies de aves y su contribución al promedio de similitud de Bray-Curtis (SIM) entre transectos, dentro de tres parches de $P$. quadrijuga en el Páramo de La Rusia (Duitama, Boyacá - Colombia), según análisis SIMPER. Se resaltan en negrita las especies con mayor contribución a las disimilitudes entre parches, y con subrayado las especies exclusivas a un solo bosque. Se utilizaron similitudes de Bray-Curtis sobre abundancias no transformadas de todas las especies encontradas en cada parche en abundancias no transformadas de todas las especies encontradas en cada parche en
cada época. Los transectos en los que no se detectó algún individuo no fueron incluidos en este análisis.

\begin{tabular}{|c|c|c|c|c|c|}
\hline \multicolumn{2}{|l|}{ Parche 1} & \multicolumn{2}{|c|}{ Parche 2} & \multicolumn{2}{|l|}{ Parche 3} \\
\hline TAXON & SIM & TAXON & SIM & TAXON & SIM \\
\hline Aglaectis cupreopennis & 29,6 & Carduelis & 53,1 & Eriocnemis vestitus & 22,7 \\
\hline Carduelis spinescens & 25,4 & spinescens & & Myioborus ornatus & 16,2 \\
\hline Notiochelidon murina & 22,9 & $\underline{\text { Grallaria rufula }}$ & 24,5 & Cinnycerthia unirufa & 16,1 \\
\hline & & & & Turdus fuscater & 14,8 \\
\hline & & & & Mecocerculus leucophrys & 11,5 \\
\hline Total & 77,9 & Total & 77,6 & Total & 81,3 \\
\hline
\end{tabular}

Con el análisis SIMPER, se detectaron tres especies para el parche 1 , dos para el parche 2 y cinco para el parche 3 , que contribuyeron con cerca del $80 \%$ de la similitud observada entre muestras de cada uno de los parche de bosque. De ellas, solo Carduelis spinescens fue característica de los parche 1 y 2 , mientras que tres especies fueron exclusivas a alguno de los parches. En este sentido, las especies con mayor contribución y dominancia para la discriminación de los parches fueron las numéricamente dominantes, así: Notiochelidon murina para el parche 1, Carduelis spinescens para el parche 2 y Cinnycerthia unirufa para el parche 3 (Tabla 2).

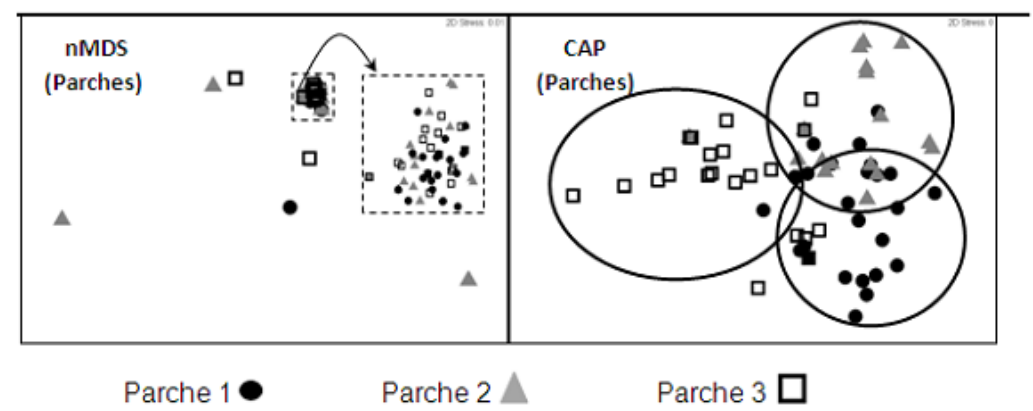

Figura 2. Estructura de los ensamblajes de aves presentes en parches de $P$. quadrijuga en el Páramo de La Rusia (Duitama, Boyacá - Colombia), según ordenaciones nMDS y CAP. Se utilizaron las similitudes de Bray-Curtis sobre abundancias no transformadas de todas las especies encontradas en 30 unidades muestrales en cada uno de los parches analizados. 


\section{DISCUSIÓN}

La riqueza de especies de aves hallada en este estudio (34 spp) concuerda con los resultados reportados por otros autores como Balderrama y Ramírez (2001); Herzog et al. (2002); Servat, Mendoza y Ochoa, (2002); Herzog et al. (2003); Ferro et al. (2006) (Tabla 3), para bosques con predominio de Polylepis, en los cuales se registran entre 34 y 60 especies de aves incluyendo algunas especies raras de avifauna altoandina para parches de Polylepis de reducida extensión. El número de especies presentes en este estudio muestra que áreas pequeñas de bosque en zonas de páramo contribuyen de manera importante a la diversidad de la avifauna.

Entre las familias de aves más comunes que utilizan los parches de Polylepis del norte de los Andes se encuentran Trochilidae, Emberizida y Tyrannidae (Balderrama \& Ramírez, 2001; Herzog et al., 2002; Servat et al., 2002; Herzog et al., 2003; Ferro et al., 2006; Cofré, 2007; Bellis et al., 2009); en cuanto a especies, se encuentran Phrygilus unicolor, Metallura tyrianthina, Ampelion rubrocristatus y Mecocerculus leucophrys (Balderrama \& Ramírez, 2001; Servat et al., 2002; Herzog et al., 2003; Bellis et al., 2009). Estos resultados confirman la presencia de algunas familias y especies de aves en los bosques de Polylepis en Colombia y registra otras especies para los bosques de $P$. quadrijuga de la cordillera Oriental en el departamento de Boyacá tales como Grallaria rufula, Margarornis squamiger, Ramphomicron microrhynchum, entre otras. 
Tabla 3. Riqueza avifaunística en diferentes estudios andinos sobre parches de Polylepis, situados por encima de $3350 \mathrm{msnm}$

\begin{tabular}{|c|c|c|c|c|}
\hline Localidad & $\begin{array}{c}\text { No. de } \\
\text { especies de } \\
\text { Polylepis }\end{array}$ & $\begin{array}{l}\text { Altitud } \\
(\mathrm{msnm})\end{array}$ & $\begin{array}{l}\text { Días de } \\
\text { trabajo }\end{array}$ & $\begin{array}{c}\text { No. } \\
\text { especies }\end{array}$ \\
\hline $\begin{array}{l}\text { Abra Málaga } \\
\text { (Ferro et al., 2006) }\end{array}$ & 2 & $4227-4400$ & 3 & 36 \\
\hline $\begin{array}{l}\text { Choquechaca } \\
\text { (Ferro et al., 2006) }\end{array}$ & 2 & $3800-4900$ & 5 & 39 \\
\hline $\begin{array}{l}\text { Cancha Cancha } \\
\text { (Ferro et al., 2006) }\end{array}$ & 2 & $4010-4850$ & 3 & 45 \\
\hline $\begin{array}{l}\text { Quishurani } \\
\text { (Ferro et al., 2006) }\end{array}$ & 2 & $4066-4700$ & 3 & 34 \\
\hline $\begin{array}{l}\text { Huilloc - Queuñacocha } \\
\text { (Ferro et al., 2006) }\end{array}$ & 2 & $4200-4800$ & 4 & 42 \\
\hline $\begin{array}{l}\text { Sacha Loma, Bolivia } \\
\text { (Herzog et al., 2003) }\end{array}$ & & $3700-3800$ & 60 & 35 \\
\hline $\begin{array}{l}\text { Sacha Loma, Bolivia } \\
\text { (Herzog et al., 2002) }\end{array}$ & 1 & $3710-3880$ & 32 & 30 \\
\hline $\begin{array}{l}\text { Parque Nacional Tunari } \\
\text { (Cochabamba, Bolivia) } \\
\text { (Balderrama \& Ramírez, } \\
\text { 2001) }\end{array}$ & 1 & $3350-4000$ & 60 & 53 \\
\hline $\begin{array}{l}\text { Sacsamonte, Yanacocha, } \\
\text { Pumahuanca, Queuñamonte } \\
\text { Cordillera Vilcabamba, Cusco } \\
\text { - Perú } \\
\text { (Servat et al., 2002) }\end{array}$ & 2 & $4050-4150$ & 16 & 43 \\
\hline $\begin{array}{l}\text { Yanacocha, Cordillera } \\
\text { Vilcabamba, Cusco - Perú } \\
\text { (Servat et al., 2002) }\end{array}$ & 2 & $3800-4200$ & 16 & 60 \\
\hline $\begin{array}{l}\text { Pumahuanca, Cordillera } \\
\text { Vilcabamba, Cusco - Perú } \\
\text { (Servat et al., 2002) }\end{array}$ & 2 & 4110 & 16 & 37 \\
\hline $\begin{array}{l}\text { Queuñamonte, Cordillera } \\
\text { Vilcabamba, Cusco - Perú } \\
\text { (Servat et al., 2002) }\end{array}$ & 2 & 3812 & 16 & 35 \\
\hline $\begin{array}{l}\text { Páramo de La Rusia (este } \\
\text { estudio) }\end{array}$ & 1 & $3612-3776$ & 30 & 34 \\
\hline
\end{tabular}

La avifauna de los parches de Polylepis del Páramo de La Rusia está integrada por especies de distribución restringida como Grallaria rufula, Leptasthenura andicola, Phrygilus unicolor y especies casi endémicas de Colombia como Myioborus ornatus, Catamenia homochroa y Eriocnemis mosquera de acuerdo a la clasificación de Stiles (1998). Así mismo, se encontraron especies endémicas para el norte de los Andes (Parker, Stotz \& Fitzpatrick, 1996) como Gallinago nobilis, Eriocnemis mosquera, E. vestitus, Lipaugus fuscocinereus, Cinnycerthia unirufa, Diglossa humeralis, D. lafresnayii, Myioborus ornatus, Conirostrum rufum y Carduelis spinescens.

La similitud en el número de especies encontrada en los parches de bosque estudiados puede estar relacionada con la heterogeneidad vegetal de cada uno de ellos. Estos parches conforman un ecotono en donde se mezclan especies vegetales de páramo con especies del borde superior del Bosque Altoandino, generando hábitats disponibles para las especies de aves, incrementando su diversidad. Harris (1988), Yanhner (1988) y Lehmkul (1990) consideran que las zonas de transición entre diferentes tipos de vegetación, sean bordes o áreas perturbadas del bosque, son beneficiosas para la fauna porque generalmente se incrementa la diversidad de especies. 
Notiochelidon murina, Cardualis spinescens y Cinnycerthia unirufa, fueron las especies que presentaron la mayor abundancia en los parches 1,2 y 3 , respectivamente. Según Hilty y Brown (1986) estas especies no tienen un rango de distribución restringido y, si bien, no son cosmopolitas, tampoco son endémicas de alguna región; razón por la cual se registraron como las más abundantes en este estudio. Fjeldså y Kessler (2004) también mencionan a las mismas especies presentes en los bosques de Polylepis.

Grallaria rufula, especie poco abundante, solo se reportó en el parche 2. Esta especie vive principalmente en el suelo y prefiere los hábitats conservados con sotobosque, características observadas en el parche 2. Kattan (1992) menciona que las especies más raras o con rango de distribución restringido siempre tienden a tener una baja abundancia debido a que presentan una alta especificidad de hábitat.

Los análisis nMDS y CAP no mostraron diferencias en cuanto a épocas climáticas. Esto se debe a que las especies encontradas son residentes, por lo tanto permanecen en el páramo a lo largo de todo el año. Herzog et al. (2003) realizaron un estudio en el que evaluaron las variables estacionales en una comunidad de aves en bosques de Polylepis besseri en Bolivia, ellos no encontraron variaciones significativas entre las épocas climáticas.

Por el contrario, el CAP identificó patrones de distribución entre los parches de bosque, gracias a que construye la ordenación basado en índices de distancia o disimilaridad (Anderson \& Willis, 2003). Cada parche de bosque presenta un ensamblaje particular que está determinado por las especies no comunes (Figura 2 - CAP), 8 especies restringidas al parche 3 y 5 especies en cada uno de los parches 1 y 2 , lo que aumenta la diversidad beta del páramo. Esto indica que áreas pequeñas de vegetación azonal de bosque en medio de las unidades de vegetación típica de páramo, funcionan como islas, cada una de las cuales posee un ensamblaje particular de aves, condicionado por pequeñas diferencias estructurales de bosque, con especies vegetales exclusivas en cada uno de ellos (Meneses \& González, 2008) y por la proximidad de otros tipos de hábitat, lo que permite ampliar o disminuir las ofertas alimentaria y de hábitat para la fauna, de acuerdo a las asociaciones vegetales presentes.

Este estudio muestra que la pérdida de pequeñas áreas de bosque, seguramente afectarán la diversidad de aves en el páramo. Los bosques de Polylepis son ecosistemas frágiles y la perdida de estos afecta la diversidad de la fauna (Fjeldså \& Kessler, 2004; Gareca et al., 2010).

No todos los fragmentos de bosque son iguales en cuanto a su estructura vegetal (Jaña-Prado et al., 2006), esta heterogeneidad de hábitat aumenta la oferta de refugio, alimento y sitios de anidación, lo que contribuye a la diversidad 
biológica (Williams, 1964), con lo que se pretende, se generen estrategias que conlleven a la conservación de los relictos de bosque de Polylepis en Colombia y la fauna asociada.

\section{AGRADECIMIENTOS}

Este trabajo se realizó en el marco del proyecto "Zonificación ambiental del corredor de páramo y bosque Alto Andino Iguaque - La Rusia - Guantiva en los departamentos de Boyacá y Santander", desarrollado por los Grupos de Investigación Manejo Integrado de Ecosistemas y Biodiversidad (XIUÂ) y Geomática y Ambiente (GIGA). Fue financiado por la Comunidad Económica Europea (CEE) a través del Instituto Agustín Codazzi (IGAC), la Universidad Pedagógica y Tecnológica de Colombia (UPTC-DIN) y la Corporación Autónoma Regional de Boyacá (CORPOBOYACÁ) (Contrato de Subvención UPTC-IGAC 1964 de 2006 y Convenio UPTCCORPOBOYACÁ 104-2006).

\section{BIBLIOGRAFÍA}

- Anderson, M. J. (2004). CAP: a FORTRAN computer program for canonical analysis of principal coordinates. New Zealand: Department of Statistics, University of Auckland.

- Anderson, M. J. y Robinson, J. (2003). Generalized discriminant analysis based on distances. Australian \& New Zealand Journal of Statistics, 45(3), 301-318.

- Anderson, M. J. y Willis, T. J. (2003). Canonical analysis of principal coordinates: a useful method of constrained ordination for ecology. Ecology, 84(2), 511-525.

- Balderrama, J. y Ramírez, M. (2001). Diversidad y endemismo de aves en dos fragmentos de bosque de Polylepis besseri en el Parque Nacional Tunari (Cochabamba, Bolivia). Revista Boliviana de Ecología, 9, 45-60.

- Bellis, L. M.; Rivera, L.; Politi, N.; Martín, E.; Perasso, L.; Cornell, F. y Renison, D. (2009). Latitudinal patterns of bird richness, diversity and abundance in Polylepis australis mountain forest of Argentina. Bird Conservation International, 19, 265-276.

- Bray, J. R. y Curtis, J. T. (1957). An ordination of the upland forest communities of southern Wisconsin. Ecological Monographs, 27(4), 325-349.

- Clarke, K. R. (1993). Non-parametric multivariate analyses of changes in community structure. Australian journal of ecology, 18(1), 117-143.

- Cofré, H. L. (2007). Avifauna asociada a los bosques de Queñoa (Polylepis spp.) del norte de Chile. Boletín Chileno de Ornitología, 13, 56-60. 
- Colwell, R. K. y Coddington, J. A. (1994). Estimating terrestrial biodiversity through extrapolation. Philosophical Transactions of the Royal Society of London. Series B: Biological Sciences, 345(1311), 101118.

- Donegan, T.; Quevedo, A.; Salaman, P. y McMullen, M. (2012). Revision of the status of bird species occurring or reported in Colombia 2012. Conservación Colombiana, 17, 4-14.

- Etter, A. y Villa, A. (2000). Andean forests and farming systems in part of the Eastern Cordillera (Colombia). Mountain Research and Development, 20, 236-245.

- Ferro, G.; Aucca, C.; Miranda, D.; Santander, O.; Valdez, Y. y Factor, F. (2006). Evaluación Ornitológica Rápida en los Bosques de Polylepis de la Cordillera del Vilcanota. Asociación Ecosistemas Andinos ECOAN.

- Fjeldså, J. (2002). Polylepis forests-vestiges of a vanishing ecosystem in the Andes. Ecotropica, 8(2), 111-123.

- Fjeldså, J. y Kessler, M. (2004). Conservación de la biodiversidad de los bosques be Polylepis de las tierras altas de Bolivia: Una contribución al manejo sustantable en los Andes (Vol. 11). Centro para la Investigación de la Diversidad Cultural y Biológica de los Bosques Pluviales Andinos (DIVA).

- Fjeldså, J.; Kessler, M.; Engblom, G. y Driesch, P. (1996). Conserving the biological diversity of Polylepis woodlands of the highland of Peru and Bolivia: a contribution to sustainable natural resource management in the Andes. Copenhagen: NORDECO.

- Fjeldså, J.; Lambin, E. y Mertens, B. (1999). Correlation between endemism and local ecoclimatic stability documented by comparing Andean bird distributions and remotely sensed land surface data. Ecography, 22, 63-78.

- Fundación PRO-AVES y American Bird Conservancy. (2007). Esperanza para los bosques de Polylepis. Lineamientos estratégicos para la Conservación de los Bosques de Polylepis en Colombia. Fundación PROAVES.

- Gareca, E.; Hermy, M.; Fjeldså, J. y Honnay, O. (2010). Polylepis woodland remnants as biodiversity islands in the Bolivian high Andes. Biodiversity and conservation, 19(12), 3327-3346.

- Harris, L. D. (1988). Edge effects and conservation of biotic diversity. Conservation Biology, 2(4), 330-332.

- Herzog, S. K.; Soria A., R. y Matthysen, E. (2003). Seasonal variation in avian community composition in a high-Andean Polylepis (Rosaceae) forest fragment. The Wilson Bulletin, 115(4), 438-447.

- Herzog, S. K.; Soria, R.; Troncoso, A. y Matthysen, E. (2002). Composition and structure of avian mixedspecies flocks in a high-Andean Polylepis forest in Bolivia. Ecotropica, 8, 133-143.

- Hilty, S. L. y Brown, B. (1986). A guide to the birds of Colombia. Princeton University Press. 
- Hjarsen, T. (1997). The effects of plantations in the Andes. Tropical Forests Update, 7, 15.

- Instituto de Hidrología, Meteorología y Estudios Ambientales (IDEAM). 2007. Sistema de Información Nacional Ambiental. Estación 2403084 Antena TV Rusia. Fecha de proceso: 14-03/2008.

- Jaña-Prado, R.; Celis-Diez, J.; Gutiérrez, A.; Cornelius, C.; Armesto, J. J.; Grez, A.; Simonetti, J. A. y Bustamante, R. O. (2006). Diversidad en bosques fragmentados de Chiloé: ¿Son todos los fragmentos iguales? In A. A. Grez, J. A. Simonetti y R. O. Bustamante (Eds.), Biodiversidad en ambientes fragmentados de Chile: Patrones y procesos a diferentes escalas (pp. 159-190). Santiago, Chile: Editorial Universitaria.

- Kattan, G. H. (1992). Rarity and vulnerability: the birds of the Cordillera Central of Colombia. Conservation Biology, 6(1), 64-70.

- Kerr, M. S. (2004). A phylogenetic and biogeographic analysis of Sanguisorbeae (Rosaceae), with emphasis on the Pleistocene radiation of the high Andean genus Polylepis. Thesis (Ph. D.). University of Maryland, College Park.

- Kessler, M. (2006). Bosques de Polylepis. Botánica Económica de los Andes Centrales, 110-120.

- Kruskal, J. B. (1964). Multidimensional scaling by optimizing goodness of fit to a nonmetric hypothesis. Psychometrika, 29(1), 1-27.

- Lehmkul, J. F. (1990). The effects of forest fragmentation on vertebrate communities in Western Oregon and Washington. Northwest environmental journal, 6(2), 433-434.

- Meneses, L. A. y González, D. A. (2008). Relación entre la diversidad de Avifauna y la estructura y composición florística de los bosques de Polylepis quadrijuga del Páramo de la Rusia, Duitama (Boyacá-Colombia). Tesis de Pregrado no publicada. Universidad Pedagógica y Tecnológica de Colombia, Tunja (Boyacá).

- Navarro, G.; Molina, J. y De la Barra, N. (2005). Classification of the high-Andean Polylepis forests in Bolivia. Plant Ecology, 176, 113-130.

- Parker, T. A.; Stotz, D. F. y Fitzpatrick, J. W. (1996). Ecological and Distributional database for Neotropical Birds. Chicago: Chicago University Press.

- Ralph, C. J.; Geupel, G. R.; Pyle, P.; Martin, T. E.; DeSante, D. F. y Milá, B. (1996). Manual de métodos de campo para el monitoreo de aves terrestres. US Department of Agriculture, Forest Service, Pacific Southwest Research Station Albanye California California.

- Rangel-Ch., J. (2000). Colombia. Diversidad Biótica III. La región de vida paramuna. Bogotá: Universidad Nacional de Colombia.

- Rangel-Ch., J. y Arellano, H. (2006). Los Bosques de Polylepis en Colombia: Riqueza y Amenazas para su Conservación. En Fundación ProAves Colombia y 
American Bird Conservancy (Ed.), Esperanza para los bosques de Polylepis. Lineamientos estratégicos para la Conservación de los Bosques de Polylepis en Colombia. Instituto de Ciencias Naturales, Universidad Nacional de Colombia.

- Salaman, P.; Donegan, T. y Caro, D. (2009). Listado de Aves de Colombia. Conservación Colombiana, 8, 2-89.

- Servat, G. P.; Mendoza, W. y Ochoa, J. A. (2002). Flora y fauna de cuatro bosques de Polylepis (Rosaceae) en la Cordillera del Vilcanota (Cusco, Perú). Ecología Aplicada, 1(1), 25-35.

- Stiles, F. G. (1998). Aves endémicas de Colombia. En Instituto de Investigaciones de Recursos Biológicos Alexander von Humbolt, PNUMA \& Ministerio de Medio Ambiente (Eds.), Informe Nacional sobre el Estado de la Biodiversidad en Colombia (Vol. 1, pp. 378-432). Bogotá.

- Stiles, F. G. y Bohórquez, C. I. (2000). Evaluando el estado de la Biodiversidad: el caso de la avifauna de la Serranía de las Quinchas, Boyacá, Colombia. Caldasia, 22(1), 61-92.

- Stiles, F. G. y Rosselli, L. (1998). Inventario de las aves de un bosque altoandino: comparación de dos métodos. Caldasia, 20(1), 29-43.

- Tarifa, T. y Yensen, E. (2001). Mamíferos de los bosques de Polylepis en Bolivia. Revista Boliviana de Ecología y Conservación Ambiental, 9, 29-44.

- Valderrama, S. V. y Verhelst, J. C. (2006). Avifauna asociada a bosques de Polylepis en Colombia. En Fundación Pro-Aves Colombia y American Bird Conservancy (Eds.), Esperanza para los bosques de Polylepis. Lineamientos estratégicos para la Conservación de los Bosques de Polylepis en Colombia. Instituto de Ciencias Naturales, Universidad Nacional de Colombia.

- Venero, J. y Macedo, H. D. (1983). Relictos de bosques en la puna del Perú. Boletín de Lima, 30, 19-26.

- Warwick, R.; Clarke, K. y Saharsono. (1990). A statistical analysis of coral community responses to the 1982-83 El Niño in the Thousand Islands, Indonesia. Coral Reefs, 8(4), 171-179.

- Williams, C. (1964). Patterns in the balance of nature. New York: Academic Press.

- Yahner, R. H. (1988). Changes in Wildlife Communities Near Edges. Conservation Biology, 2(4), 333-339.

1. Bióloga, Especialista en Educación Ambiental. Investigador Grupo de Investigación Manejo Integrado de Ecosistemas y Biodiversidad - XIUÂ. Escuela de Ciencias Biológicas, Universidad Pedagógica y Tecnológica de Colombia. Dirección: Escuela de Ciencias Biológicas, Universidad Pedagógica y Tecnológica de Colombia -UPTC-, Avenida Central del 
Norte, Tunja-Boyacá, Colombia.

andreitabiologa@gmail.com

2. Biólogo, M.Sc. en Ciencias Biológicas. Grupo de Investigación Manejo Integrado de Ecosistemas y Biodiversidad - XIUÂ. Escuela de Ciencias Biológicas, Universidad Pedagógica y Tecnológica de Colombia. Dirección: Escuela de Ciencias Biológicas, Universidad Pedagógica y Tecnológica de Colombia -UPTC-, Avenida Central del Norte, Tunja-Boyacá, Colombia. yimyherrera@yahoo.com 